\title{
BMJ Open Resting-state functional reorganisation in Alzheimer's disease and amnestic mild cognitive impairment: protocol for a systematic review and meta-analysis
}

\author{
Diyang Lyu, ${ }^{1}$ Taoran Li, ${ }^{1}$ Xuanxin Lyu (D) ${ }^{2}$
}

To cite: Lyu D, Li T, Lyu X. Resting-state functional reorganisation in Alzheimer's disease and amnestic mild cognitive impairment: protocol for a systematic review and meta-analysis. BMJ Open 2021;11:e049798. doi:10.1136/ bmjopen-2021-049798

- Prepublication history and additional supplemental material for this paper are available online. To view these files, please visit the journal online (http://dx.doi.org/10.1136/ bmjopen-2021-049798).

DL and TL contributed equally.

Received 04 February 2021 Accepted 29 September 2021

Check for updates

(c) Author(s) (or their employer(s)) 2021. Re-use permitted under CC BY-NC. No commercial re-use. See rights and permissions. Published by BMJ.

${ }^{1}$ Capital Medical University, Beijing, China

${ }^{2}$ Neurological Rehabilitation Center, Beijing Rehabilitation Hospital, Capital Medical University, Beijing, China

Correspondence to

Dr Xuanxin Lyu;

Ivxvanxin@163.com

\section{ABSTRACT}

Introduction The incidence of Alzheimer's disease $(A D)$ is increasing rapidly, causing a growing burden to health and economic worldwide. Several clinical trials in the past decade failed to find solutions, and there remains a lack of an effective treatment. The evidence suggests that early intervention for neurodegeneration would likely be effective in preventing cognitive decline. Cognitive decline in AD occurs continuously over a long period; however, there remains a lack of simple, rapid and accurate approach for diagnosis of amnestic mild cognitive impairment or subjective cognitive decline due to underlying Alzheimer's pathology. Resting-state functional MRI (rs-fMRI) determines the functional activities of the human brain non-invasively. The amplitude of lowfrequency fluctuation (ALFF), fractional ALFF (fALFF) and regional homogeneity ( $\mathrm{ReHo}$ ) are rs-fMRI indicators with high repeatability. They have been studied as early diagnostic imaging markers for other diseases and may be promising markers also for $\mathrm{AD}$.

Methods and analysis The following electronic literature databases will be searched from inception to December 2021: Medline-Ovid, Medline-PubMed, EMBase-Ovid, Cochrane Central and ClinicalTrials.gov. Two independent reviewers will select studies with eligible criteria, extract data and assess the quality of the original studies with our quality assessment tool individually. Missing data will be requested by sending emails to the corresponding authors. Brain regions will be presented for ALFF/fALFF and $\mathrm{ReHo}$ by performing activation likelihood estimation with the Seed-based $d$ Mapping-Permutation of subject images V.6.21 software. Meta-regression will be performed to determine the potential brain regions that may strongly correlate with cognitive decline progression. Subgroup analysis, funnel plot, Egger's test and sensitivity analysis will be conducted to detect and explain potential heterogeneity.

Ethics and dissemination This study does not require formal ethical approval. The findings will be submitted to a peer-review journal.

PROSPERO registration number CRD42021229009.

\section{INTRODUCTION}

Alzheimer's disease (AD) is a neurodegenerative disease that is causing a growing burden on health and economic. ${ }^{1} \mathrm{AD}$ will
Strengths and limitations of this study

- This systematic review and meta-analysis will summarise brain regions with amplitude of lowfrequency fluctuation (ALFF)/fractional ALFF or regional homogeneity alterations in Alzheimer's disease (AD) and amnestic mild cognitive impairment (aMCl) patients using qualitative and quantitative analyses.

- This study will consider $\mathrm{AD}$ and $\mathrm{aMCl}$ as different stages of cognitive decline and conduct a metaregression analysis with the pooled population to explore the brain regions closely related to the stages of cognitive decline.

- We established a modified quality assessment tool to assess the quality of the original studies for resting-state functional MRI systematic review and meta-analysis.

- We will only retrieve data from English-language databases and may overlook a few valuable original studies in other languages.

reportedly affect 131 million people worldwide by 2050 and will cause over US\$2 trillion in economic losses by $2030 .{ }^{2}$ However, a satisfactory therapeutic breakthrough in the field of AD is still lacking. Since the approval of memantine in $2003,{ }^{3}$ all clinical trials of new drugs developed for the different pathogeneses of $\mathrm{AD}$ have failed to improve clinical outcome. ${ }^{4}$ The molecular targeted therapies can reduce the pathological products of $\mathrm{AD}$ in the human brain but it is yet uncertain if they are effective in $\mathrm{AD}{ }^{5}$ The failure of such therapies has been attributed to the fact that most of the study participants were patients with moderate or severe AD. These patients exhibit pathological changes in the brain, which may be irreversible or challenging to stop the progression, and the optimal time for treatment may have already elapsed. ${ }^{67}$ Thus, the early diagnosis and treatment of patients with $\mathrm{AD}$ may be the key to halt the disease progression. In the Alzheimer's continuum, 
patients in the predementia stages may exhibit clinical symptoms difficult to identify, such as mild cognitive impairment (MCI) or subjective cognitive decline (SCD). Efficiently and accurately differentiating them from cognitively healthy individuals through clinical information and neuropsychological scales is complicated, especially for clinicians who are not experts in the AD field. ${ }^{8}$ Therefore, there is currently a high demand for convenient and reliable markers for the diagnosis of $\mathrm{AD}{ }^{9}$

The accurate diagnosis of $\mathrm{AD}$ is usually confirmed by autopsy; however, in the recent decade, with the development of laboratory tests and neuroimaging, scientists can directly detect biomarkers in vivo to identify patients with AD. ${ }^{9-11}$ These biomarkers include detection of amyloid- $\beta$ and hyperphosphorylated tau, found in the plasma, cerebrospinal fluid ${ }^{6}$ and with positron emission tomography imaging. ${ }^{12}$ However, interlaboratory variations lead to a lack of robustness of these biomarkers for early diagnosis. Although several other biomarkers have been proposed, repeated validation is still required to prove their reliability. ${ }^{1314}$ In addition, these examinations can be harmful through vascular punctures, lumbar punctures or the administration of radioactive substances. Conversely, MRI can non-invasively visualise structural and functional changes in the brain. The current diagnostic criteria are mainly based on the structural MRI reports of atrophy of the whole brain, medial temporal lobe or hippocampus, and other brain alterations. ${ }^{12}$ However, functional MRI (fMRI) may reflect the brain's functional state through the changes in cerebral blood-oxygen signal, detect brain abnormalities before detectable structural changes, and indicate risk of cognitive decline.

Resting-state fMRI (rs-fMRI) is a non-invasive, harmless and efficient imaging detection method with high spatial resolution, showing the functional status of the central nervous system. Since 1995, rs-fMRI has been increasingly used in scientific research. ${ }^{15}$ Several indicators of rs-fMRI have been used to reflect functional activity, including functional connectivity ${ }^{16}$ the amplitude of low-frequency fluctuation (ALFF), ${ }^{17}$ fractional ALFF (fALFF) ${ }^{18}$ and regional homogeneity (ReHo) ${ }^{19}$ Among the commonly used indicators, ALFF/fALFF and ReHo are reported to have relatively higher test-retest reliability than other biomarkers, ${ }^{20}$ and the calculation process does not require prior assumptions of the specific brain regions to be studied. ALFF is considered to represent spontaneous brain activity, and fALFF is derived from an improved algorithm of ALFF. ${ }^{21}$ ReHo assesses the synchronisation among one voxel and its neighbours (eg, 26 voxels) and is considered to represent the homogeneity of a given cluster. $^{21}$ These indicators were also proven to correlate with disease progression in other diseases through general linear models or machine learning approaches and are considered potential diagnostic markers. ${ }^{22-24}$ However, fMRI is still used as an additional resource in conjunction with other tests. We speculate that ALFF/ fALFF and ReHo may be promising imaging markers for the early diagnosis of $\mathrm{AD}$.
Previous studies in the field of $\mathrm{AD}$ or amnestic MCI (aMCI) have reported several brain regions with increased or decreased ALFF/fALFF or ReHo. ${ }^{25}{ }^{26}$ However, a few studies have examined patients with $\mathrm{SCD}{ }^{27}$ Uniform diagnostic criteria for SCD was not established for decades, resulting in the heterogeneity of subjects in different studies. ${ }^{27}$ This lack of clarity may be due to the concept of SCD was introduced in recent years, ${ }^{28}$ its definition and clinical significance has not been fully clarified. Additionally, SCD is a naturally heterogeneous state and a construct that is greatly affected by psychosocial factors including cultural background. ${ }^{29}{ }^{30}$ Thus, this systematic review and meta-analysis will exclude SCDrelated studies from pooled estimations if there are not enough original studies with high quality and consistent diagnostic criteria. A previous meta-analysis summarised 12 original studies and reported that eight brain regions showed altered ALFFs in aMCI patients compared with healthy controls. ${ }^{25}$ Another meta-analysis included ten original studies of aMCI patients and reported 11 brain regions with altered ReHos in these patients compared with healthy controls. ${ }^{26}$ The above reviews were published several years ago, and their findings might change with recent original studies.

\section{Objective}

We will conduct this systematic review and meta-analysis to summarise previous rs-fMRI studies comparing patients with $\mathrm{AD}$ or aMCI to adults with normal cognition and determine the group differences in ALFF/fALFF or ReHo. SCD studies will also be included, if available. Furthermore, we will also identify the brain regions associated with the severity of the disease or cognitive decline by using meta-regression. Brain regions with increased or decreased ALFF/fALFF or ReHo will be determined through a meta-analysis, and reported for potential incorporation in clinical practice and the establishment of diagnostic criteria.

\section{METHODS AND ANALYSIS \\ Study guidelines and registration}

This systematic review and meta-analysis will include studies reporting ALFF/fALFF or ReHo in AD or aMCI patients compared with cognitively healthy controls. Thus, the Quality of Reporting of Meta-Analyses guidelines is not applicable for this study. ${ }^{31}$ The guidelines of the Preferred Reporting Items for Systematic Reviews and Meta-Analyses (PRISMA) statement were updated from the Quality of Reporting of Meta-analyses (QUOROM) statement and apply to all types of systematic reviews. ${ }^{32}$ Meta-analysis of Observational Studies in Epidemiology (MOOSE) has also been established for meta-analysis of observational studies. ${ }^{33}$ Therefore, a systematic review and meta-analysis will be conducted and presented following the PRISMA statement, ${ }^{32}$ MOOSE guidelines ${ }^{33}$ and Cochrane Handbook. ${ }^{34}$ This protocol follows the PRISMA protocols statement. ${ }^{35}$ 


\begin{tabular}{|c|c|}
\hline Search & Query \\
\hline \#1 & (alzheimer*[Title/Abstract]) OR (alzheimer disease[MeSH Terms]) \\
\hline \#3 & $\begin{array}{l}\text { (((((MCI[Title/Abstract]) OR (“mild cognitive impairment”[Title/Abstract])) OR (“amnestic mild cognitive } \\
\text { impairment”[Title/Abstract])) OR (aMCI[Title/Abstract])) OR (SCD[Title/Abstract]])) OR (“subjective cognitive } \\
\text { decline"[Title/Abstract]) }\end{array}$ \\
\hline \#5 & ((impair*[Title/Abstract]) OR (decline[Title/Abstract])) OR (reduc*[Title/Abstract]) \\
\hline \#6 & $\# 4$ and \#5 \\
\hline$\# 7$ & $\begin{array}{l}\text { ((((((“functional magnetic resonance imaging"[Title/Abstract]) OR (“magnetic resonance imaging"[Title/Abstract])) } \\
\text { OR (“resting-state functional magnetic resonance imaging"[Title/Abstract])) OR ("resting state functional magnetic } \\
\text { resonance imaging"[Title/Abstract])) OR (fMRI[Title/Abstract])) OR ("functional MRI"[Title/Abstract])) OR (MRI[Title/ } \\
\text { Abstract])) OR (“rs-fMRI"[Title/Abstract]) }\end{array}$ \\
\hline \#9 & \#1 or \#2 or \#3 or \#6 \\
\hline \#10 & $\# 7$ and \#8 and \#9 \\
\hline
\end{tabular}

\section{Search strategy}

We will search the following electronic databases from inception to December 2021 for published literature: Medline-Ovid, Medline-PubMed, EMBase-Ovid and Cochrane Central. We will also search the ClinicalTrials registration platform for missing, unpublished, or ongoing studies. Two independent reviewers (DL and TL) will also examine the reference lists of each article that qualifies for the full-text screening step and of each review article in this field. After data extraction, we will send emails to the corresponding authors of the included studies for additional information to avoid potentially missing data. The search strategy for Medline-PubMed is presented in table 1, and the full search strategy is presented in online supplemental file 1.

\section{Eligibility criteria}

Studies will be included in the systematic review and meta-analysis according to the following criteria:

- Patients: The patients enrolled in the original studies have been diagnosed with mild, moderate or severe $\mathrm{AD}$ or aMCI according to clearly reported diagnostic criteria. We will not limit the exact diagnostic criteria in the original studies, although there are some of the most commonly used criteria for $\mathrm{AD}^{912}$ or aMCI. ${ }^{36} 37$ The exact scores for cognitive assessments are preferably reported; however, a specific format is not required. We will not restrict data collection on the age, sex, or race of participants in the original studies; however, it will be reported in detail. Moreover, studies focused on AD or aMCI with other complications, such as postdementia depression, are eligible if there are clear descriptions in the literature.

- Controls: The control group will include cognitively healthy subjects with comparable demographic characteristics to the $\mathrm{AD}$ or aMCI group in the original studies.

- Outcomes: Only rs-fMRI studies will be included. The original studies should report the results from wholebrain analysis, rather than analyses of specific brain networks or regions. Brain regions with increased or decreased ALFF/fALFF or ReHo will be included in the pooled estimation through activation likelihood estimation (ALE) analysis. The outcomes will preferably be reported with the peak coordinates, cluster size, and statistics of each brain region. Additionally, only the studies which mentioned the use of multiple comparison correction will be included in the metaanalysis to avoid significant false-positive results induced by the authors, ${ }^{15}$ while all results reported in these studies will be put into pooled estimation to avoid missing important data.

- Study design: Observational studies with or without further follow-up measurements will be included. Controlled clinical trials that reported the differences between patients and controls at baseline will also be included if they meet the above criteria.

\section{Study selection}

Two independent reviewers (DL and TL) will screen the titles and abstracts of the articles found in each electronic database after removing duplications with EndNote V.X9 software. The studies that are inconsistent with our eligibility criteria will be excluded by the each reviewer individually. Then, the reviewers will examine the full text of the articles and further exclude those that do not meet our criteria. The reasons for each exclusion in this step will be recorded individually. An independent reviewer (XL) will solve any disagreement between the two reviewers, which will be recorded with detailed explanation. The 


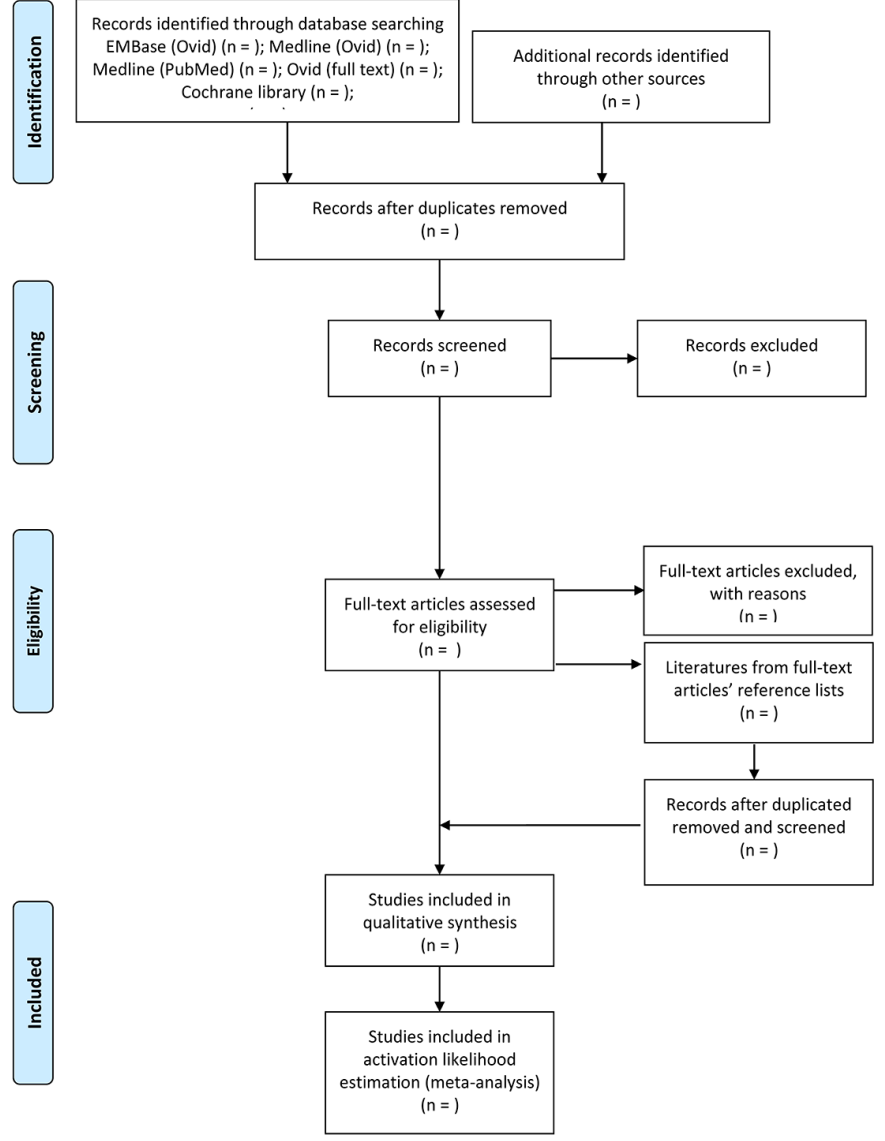

Figure 1 The PRISMA flow diagram of this systematic review and meta-analysis. PRISMA, Preferred Reporting Items for Systematic Reviews and Meta-Analyses.

complete process of the study selection will be presented in a PRISMA flow diagram (figure 1).

\section{Data extraction}

The same reviewers (DL and TL) will extract demographic information, the study design, data analysis and outcomes individually using Microsoft Excel. The complete information and data extraction lists are presented in table 2. Demographic information includes age, sex, nationality, race, apolipoprotein $\mathrm{E}$ genotype (APOE), years since first symptom or initial diagnosis, education level and cognitive assessment scores. Information about the disease stage will be extracted directly from the original articles and determined through their clinical dementia rating scores $(0.5$ for aMCI, 1 for mild AD, 2 for moderate AD and 3 for severe AD) ${ }^{38}$ Information about the study design and data analysis will include the field strength and exact machine model of the MRI scanner, the statistical method of multiple comparison correction, the software and packages used, the frequency range for ALFF/fALFF, the number of neighbouring voxels for $\mathrm{ReHo}$, and the parameter of full width at half maximum smooth kernel of each original study. The results include the anatomical labels, peak coordinates, and cluster size of each reported brain region from the original studies, and the cognitive assessment outcomes, such as the MiniMental State Examination scores. Continuous variables will be recorded as means $\pm \mathrm{SD}$, and discrete variables will be recorded in percentages. Values reported as median with range or IQR will be converted into mean \pm SD with validated algorithms. ${ }^{3940}$ The recorded data will be verified by comparing the reports of the two reviewers. In case of studies without multiple comparison correction or with missing data, we will also contact the corresponding authors as necessary.

\section{Quality assessment}

To our knowledge, there is no standard checklist or tool for the quality assessment of fMRI studies. Thus, we have developed a quality assessment tool for this study, as shown in table 3 , based on a previous meta-analysis. ${ }^{2641}$ The two independent reviewers (DL and TL) will examine the full texts for any potential bias according to our quality assessment tool and grade each original study. The total quality assessment score will be reported in the main text, and a detailed quality assessment table will be provided in our systematic review and meta-analysis.

\section{Qualitative and quantitative synthesis}

Qualitative data synthesis

First, we will create a summary table to present the characteristics of the selected studies, including the

Table 2 Data and information extraction list

\begin{tabular}{|c|c|}
\hline Item & Content \\
\hline $\begin{array}{l}\text { Publication } \\
\text { information }\end{array}$ & Authors, publish year, email of the corresponding author, country. \\
\hline Data acquisition & $\begin{array}{l}\text { The field strength and the exact machine model of the MRI scanner, slice numbers and thickness of each } \\
\text { sequence, time points and total scanning time of functional MRI. }\end{array}$ \\
\hline Data processing & $\begin{array}{l}\text { Software used, number of time points to be discarded, resampling parameters, full-width at half-maximum } \\
\text { smooth kernel size, variables to be regressed out, the frequency range for ALFF/fALFF and the number of } \\
\text { neighbouring voxels for ReHo, method to control false positive rate. }\end{array}$ \\
\hline
\end{tabular}

ALFF, amplitude of low frequency fluctuation; APOE, apolipoprotein E; fALFF, fractional ALFF; ReHo, regional homogeneity. 
Table 3 Quality assessment tool of original $\mathrm{fMRI}$ studies

\section{Category 1: sample characteristics (10)}

1 Participants were enrolled with clearly described standardised diagnostic criteria (2).

2 Comprehensive demographic data with comparable baseline between groups (1).

3 Inclusion and exclusion criteria are reasonable, taking into account the possible affecting factors (2).

4 Cognitive outcomes were reported in detail (3).

$5 \quad$ Sample size $>10$ in each group (2).

\section{Category 2: methodology and reporting (10)}

6 The machine model and field strength of the MRI scanner is reported (1).

7 A clear description of the study procedure and quality control, includes the methods to ensure that the subjects are in a 'resting state' (2).

8 At least 5 minutes of resting state acquisition (2).

9 The report of scanning parameters is comprehensive and reasonable, and the quality control method of image scanning is reported (1).

\begin{tabular}{|c|c|}
\hline 10 & $\begin{array}{l}\text { Detailed description of software and toolkits used } \\
\text { (1). }\end{array}$ \\
\hline 11 & $\begin{array}{l}\text { Results were applied and reported in original } \\
\text { literature, including peak coordinate and cluster } \\
\text { size of each brain region (1). }\end{array}$ \\
\hline 12 & $\begin{array}{l}\text { Multiple comparison corrections were applied and } \\
\text { reported in the original literature (1). }\end{array}$ \\
\hline 13 & $\begin{array}{l}\text { Conclusions were consistent with the results } \\
\text { obtained and the limitations were discussed (1). }\end{array}$ \\
\hline
\end{tabular}

MRI, Magnetic resonance imaging.

information to be extracted, such as the publication year, demographic characteristics of participants, study design, analysis parameters, and outcome indicators, as shown in table 2. Then, we will provide a general summary of the study outcomes.

\section{Quantitative data synthesis}

We will conduct a quantitative analysis of each outcome. First, all brain coordinates will be converted to their corresponding Montreal Neurological Institute (MNI) space. Then, we will perform an ALE meta-analysis for ALFF/fALFF and ReHo. The pooled estimations will reveal consistent brain regions with significant functional alterations reported from different studies using the Seed-based $d$ Mapping-Permutation of subject images (SDM-PSI) V.6.21 software (www.sdmproject.com). ${ }^{42}$ The SDM-PSI algorithm is updated using the ALE algorithm. Compared with the software using the ALE algorithm, SDM-PSI can retain positive and negative activation results and avoid bias due to overlapping activation positions in the brain. The peak coordinates in the MNI space and their effect sizes (eg, t-values) extracted from original studies will be registered as centres in the 3D Gaussian probability distribution to recreate standard MNI brain maps for each study. ${ }^{43}$ Subsequently, the brain maps will be used to generate pooled estimations for each outcome separately. The sample size, sex, age and other demographic information of each group will also be used to achieve a linear mixed model analysis to control the effect of potential confounding factors. We will perform correlation analyses or partial correlation analyses to exclude variables with strong collinearity. Finally, a principal component analysis will be performed to extract several principal components as necessary; the principal components will be used as variables. The above analyses will be performed with R V.4.0.3. We will also employ a familywise error correction to control the false-positive rate.

We will further analyse the results with subgroup analyses to detect potential heterogeneity and explain its possible reasons. The data from different disease stages (AD or aMCI), with or without complications, and MRI scanners with different field strengths $(1.5 \mathrm{~T}$ or $3.0 \mathrm{~T})$ will be analysed separately. After the subgroup analyses, we will perform a sensitivity analysis by excluding studies one by one to determine whether the pooled estimations are stable or not. In this step, significant changes may indicate significant heterogeneity among the studies. Significant heterogeneity will be reported on occurrence. We will apply funnel plots to detect potential reporting biases, when no less than ten original studies are pooled in a meta-analysis. ${ }^{45}$ We will attempt to identify possible reasons and interpret existing publication biases. We will perform a meta-regression to identify potential factors that may contribute to the stage-specific changes in different brain regions, we will perform a meta-regression. In this step, we will put all included studies into pooled estimation. The following variables will be analysed: age, sex, cognitive assessment score and $A P O E$ genotype. We will perform a meta-regression using the SDM-PSI software V.6.21. We will contact the corresponding authors via email To retrieve missing data; if these values are unavailable, we will remove the related regression factors or the original study.

\section{Patient and public involvement}

As this is a protocol for a systematic review and metaanalysis, we will obtain data from published literature or the corresponding authors. Thus, patients or the public will not be involved.

\section{ETHICS AND DISSEMINATION}

This study does not require any formal ethical approval. The findings will be submitted for publication in a peerreviewed journal.

\section{DISCUSSION}

This systematic review will comprehensively summarise and analyse the results of previous fMRI studies 
investigating AD or aMCI using ALFF/fALFF or ReHo. We will present whole-brain ALFF/fALFF and ReHo analyses and report significant differences between patients with $\mathrm{AD}$ or aMCI and cognitively healthy controls.

A previous meta-analysis summarised 12 original studies and reported that 4 brain regions exhibited decreased ALFFs, while another four showed increased ALFFs in aMCI patients compared with healthy controls. ${ }^{25}$ The authors also reported that a greater decrease in ALFFs in the cuneus/precuneus cortex may be associated with the severity of cognitive impairment. ${ }^{25}$ Another meta-analysis included 10 original studies of patients with aMCI and focused on ReHo. ${ }^{26}$ The authors found that the ReHo of 11 brain regions from four brain networks differed between aMCI patients and healthy controls. The above meta-analyses were conducted several years ago, and their results varied. However, these studies included AD and aMCI only, and we will also include studies on SCD, if possible. Furthermore, our meta-analysis may provide different estimations by including recent studies in this field.

To our knowledge, only one previous meta-analysis investigated the functional characteristics of $\mathrm{AD}$ and aMCI patients compared with healthy controls through both ALFF/fALFF and ReHo. ${ }^{46}$ Their findings revealed that patients with aMCI and AD exhibited consistently impaired functional characteristics, and the alterations in the brain regions were relatively consistent. Although this study was published 6 years ago, the findings support our idea of combining studies of $\mathrm{AD}$ and aMCI patients, including SCD studies if possible, and attempting to determine the brain regions that have altered ALFF/ fALFF or ReHo with strong correlations with cognitive decline measured by neuropsychological scales through a meta-regression.

It is generally known that there are various limitations of fMRI including it being expensive and need of specific expert interpretation so that it can used by non-experts to applying in various clinical scenarios. However, previous studies have reported a large amount of data in this field, making it feasible for scientists to establish diagnostic tools for clinician use through data mining or machine learning. Similarly, our findings may highlight utility of fMRI as an adjunctive tool in early diagnosis of $\mathrm{AD}$ and predicting cognitive decline in addition to other diagnostic tools.

Acknowledgements We would like to thank the editors for their hard work on inviting reviewers. We really appreciate the reviewers, Dr Ruben van Eijk from UMC Utrecht, and Dr Riddhi Patira from University of Pittsburgh, for their excellent hard work. We would like to thank Dr Yan Li, Dr Yuqing Shi and Dr Weijiao Zhang for their help.

Contributors $\mathrm{DL}$ and $\mathrm{XL}$ designed this study. DL and TL developed the search strategy, established the data extraction list. DL drafted the manuscript. TL and $\mathrm{XL}$ revised the manuscript and provided methodological perspectives. $\mathrm{DL}$ and TL will search and screen literatures and perform data extraction. DL will assess the quality of included studies and conduct data analyses. All authors read and approved the final manuscript.
Funding This work was supported by the Scientific Research Fund of Beijing Rehabilitation Hospital.

Competing interests None declared.

Patient consent for publication Not applicable.

Provenance and peer review Not commissioned; externally peer reviewed.

Supplemental material This content has been supplied by the author(s). It has not been vetted by BMJ Publishing Group Limited (BMJ) and may not have been peer-reviewed. Any opinions or recommendations discussed are solely those of the author(s) and are not endorsed by BMJ. BMJ disclaims all liability and responsibility arising from any reliance placed on the content. Where the content includes any translated material, BMJ does not warrant the accuracy and reliability of the translations (including but not limited to local regulations, clinical guidelines, terminology, drug names and drug dosages), and is not responsible for any error and/or omissions arising from translation and adaptation or otherwise.

Open access This is an open access article distributed in accordance with the Creative Commons Attribution Non Commercial (CC BY-NC 4.0) license, which permits others to distribute, remix, adapt, build upon this work non-commercially, and license their derivative works on different terms, provided the original work is properly cited, appropriate credit is given, any changes made indicated, and the use is non-commercial. See: http://creativecommons.org/licenses/by-nc/4.0/.

\section{ORCID iD}

Xuanxin Lyu http://orcid.org/0000-0003-1841-6306

\section{REFERENCES}

1 GBD 2016 Dementia Collaborators. Global, regional, and national burden of Alzheimer's disease and other dementias, 1990-2016: a systematic analysis for the global burden of disease study 2016. Lancet Neurol 2019;18:88-106.

2 International AsD. World Alzheimer report 2015. the global impact of dementia: an analysis of prevalence, incidence, cost and trends. London: Alzheimer's Disease International, 2015.

3 Joe E, Ringman JM. Cognitive symptoms of Alzheimer's disease: clinical management and prevention. BMJ 2019;367:I6217.

4 Cummings J, Lee G, Ritter A. Alzheimer's disease drug development pipeline: 2020. Alzheimer's \& dementia 2020;6:e12050.

5 Lu L, Zheng X, Wang S, et al. Anti-A $\beta$ agents for mild to moderate Alzheimer's disease: systematic review and meta-analysis. J Neurol Neurosurg Psychiatry 2020;91:1316-24.

6 Blennow K, Dubois B, Fagan AM, et al. Clinical utility of cerebrospinal fluid biomarkers in the diagnosis of early Alzheimer's disease. Alzheimers Dement 2015;11:58-69.

7 Mauricio R, Benn C, Davis J, et al. Tackling gaps in developing life-changing treatments for dementia. Alzheimers Dement 2019;5:241-53.

8 Rodríguez-Gómez O, Rodrigo A, Iradier F, et al. The MOPEAD project: advancing patient engagement for the detection of "hidden" undiagnosed cases of Alzheimer's disease in the community. Alzheimers Dement 2019;15:828-39.

9 Jack CR, Bennett DA, Blennow K, et al. NIA-AA research framework: toward a biological definition of Alzheimer's disease. Alzheimers Dement 2018;14:535-62.

10 McKhann GM, Knopman DS, Chertkow H, et al. The diagnosis of dementia due to alzheimer's disease: recommendations from the national institute on aging-alzheimer's association workgroups on diagnostic guidelines for alzheimer's disease. Alzheimers Dement 2011;7:263-9.

11 Dubois B, Feldman $\mathrm{HH}$, Jacova $\mathrm{C}$, et al. Advancing research diagnostic criteria for Alzheimer's disease: the IWG-2 criteria. Lancet Neurol 2014;13:614-29.

12 Dubois B, Feldman HH, Jacova C, et al. Research criteria for the diagnosis of Alzheimer's disease: revising the NINCDS-ADRDA criteria. Lancet Neurol 2007;6:734-46.

13 Laske C, Sohrabi HR, Frost SM, et al. Innovative diagnostic tools for early detection of Alzheimer's disease. Alzheimers Dement 2015;11:561-78.

14 Ausó E, Gómez-Vicente V, Esquiva G. Biomarkers for Alzheimer's disease early diagnosis. J Pers Med 2020;10. doi:10.3390/ jpm10030114. [Epub ahead of print: 0409 2020].

15 Eklund A, Nichols TE, Knutsson $\mathrm{H}$. Cluster failure: why fMRI inferences for spatial extent have inflated false-positive rates. Proc Natl Acad Sci U S A 2016;113:7900-5.

16 Biswal B, Yetkin FZ, Haughton VM, et al. Functional connectivity in the motor cortex of resting human brain using echo-planar MRI. Magn Reson Med 1995;34:537-41. 
17 Zang Y-F, He Y, Zhu C-Z, et al. Altered baseline brain activity in children with ADHD revealed by resting-state functional MRI. Brain Dev 2007;29:83-91.

18 Zou Q-H, Zhu C-Z, Yang Y, et al. An improved approach to detection of amplitude of low-frequency fluctuation (ALFF) for resting-state fMRI: fractional ALFF. J Neurosci Methods 2008;172:137-41.

19 Zang Y, Jiang T, Lu Y, et al. Regional homogeneity approach to fMRI data analysis. Neuroimage 2004;22:394-400.

20 Chen X, Lu B, Yan C-G. Reproducibility of R-fMRI metrics on the impact of different strategies for multiple comparison correction and sample sizes. Hum Brain Mapp 2018;39:300-18.

21 Lv H, Wang Z, Tong E, et al. Resting-State functional MRI: everything that Nonexperts have always wanted to know. AJNR Am J Neuroradiol 2018;39:1390-9.

$22 \mathrm{Ji} \mathrm{L}$, Meda SA, Tamminga CA et al. Characterizing functiona regional homogeneity (ReHo) as a B-SNIP psychosis biomarker using traditional and machine learning approaches. Schizophr Res 2020;215:430-8.

23 Chen J, Yang J, Huang X, et al. Brain functional biomarkers distinguishing premature ejaculation from Anejaculation by ALFF: a resting-state fMRI study. J Sex Med 2020;17:2331-40.

$24 \mathrm{Ma} \mathrm{X,} \mathrm{Lu} \mathrm{F,} \mathrm{Hu} \mathrm{C,} \mathrm{et} \mathrm{al.} \mathrm{Dynamic} \mathrm{alterations} \mathrm{of} \mathrm{spontaneous} \mathrm{neural}$ activity in patients with amyotrophic lateral sclerosis. Brain Imaging Behav 2021;15:2101-8.

25 Pan P, Zhu L, Yu T, et al. Aberrant spontaneous low-frequency brain activity in amnestic mild cognitive impairment: a meta-analysis of resting-state fMRI studies. Ageing Res Rev 2017;35:12-21.

26 Zhen D, Xia W, Yi ZQ, et al. Alterations of brain local functional connectivity in amnestic mild cognitive impairment. Trans/ Neurodegener 2018;7:26.

27 Wang X, Huang W, Su L, et al. Neuroimaging advances regarding subjective cognitive decline in preclinical Alzheimer's disease. Mol Neurodegener 2020;15:55.

28 Jessen F, Amariglio RE, Buckley RF, et al. The characterisation of subjective cognitive decline. Lancet Neurol 2020;19:271-8.

29 Wu Q. Subjective cognitive impairment of older adults: a comparison between the US and China. Int J Methods Psychiatr Res 2016;25:68-75

30 Jackson JD, Rentz DM, Aghjayan SL, et al. Subjective cognitive concerns are associated with objective memory performance in Caucasian but not African-American persons. Age Ageing 2017;46:988-93.

31 Moher D, Cook DJ, Eastwood S, et al. Improving the quality of reports of meta-analyses of randomised controlled trials: the QUOROM statement. Quality of reporting of meta-analyses. Lancet 1999;354:1896-900.
32 Liberati A, Altman DG, Tetzlaff J, et al. The PRISMA statement for reporting systematic reviews and meta-analyses of studies that evaluate healthcare interventions: explanation and elaboration. BMJ 2009;339:b2700.

33 Stroup DF, Berlin JA, Morton SC, et al. Meta-Analysis of observational studies in epidemiology: a proposal for reporting. meta-analysis of observational studies in epidemiology (moose) group. JAMA 2000;283:2008-12.

34 Higgins JPT TJ, Chandler J, Cumpston M, eds. Cochrane Handbook for Systematic Reviews of Interventions version 6.1 (updated September 2020). The Cochrane Collaboration, 2020. www.training. cochrane.org/handbook

35 Shamseer L, Moher D, Clarke M, et al. Preferred reporting items for systematic review and meta-analysis protocols (PRISMA-P) 2015 elaboration and explanation. BMJ 2015;350:g7647.

36 Petersen RC, Smith GE, Waring SC, et al. Mild cognitive impairment: clinical characterization and outcome. Arch Neurol 1999;56:303-8.

37 Petersen RC, Parisi JE, Dickson DW, et al. Neuropathologic features of amnestic mild cognitive impairment. Arch Neurol 2006;63:665-72.

38 Morris JC. The clinical dementia rating (CDR): current version and scoring rules. Neurology 1993;43:2412-4.

39 Luo D, Wan X, Liu J, et al. Optimally estimating the sample mean from the sample size, median, mid-range, and/or mid-quartile range. Stat Methods Med Res 2018;27:1785-805.

40 Wan X, Wang W, Liu J, et al. Estimating the sample mean and standard deviation from the sample size, median, range and/or interquartile range. BMC Med Res Methodol 2014;14:135.

41 Zhong S, Hu Y, Fu Y, et al. Functional MRI in the effect of transcranial magnetic stimulation therapy for patients with schizophrenia: a metaanalysis protocol. BMJ Open 2020;10:e038557.

42 Albajes-Eizagirre A, Solanes A, Vieta E, et al. Voxel-based metaanalysis via permutation of subject images (PSI): theory and implementation for SDM. Neuroimage 2019;186:174-84.

43 Radua J, Rubia K, Canales-Rodríguez EJ, et al. Anisotropic kernels for coordinate-based meta-analyses of neuroimaging studies. Front Psychiatry 2014;5:13.

44 Lim L, Radua J, Rubia K. Gray matter abnormalities in childhood maltreatment: a voxel-wise meta-analysis. Am J Psychiatry 2014;171:854-63.

45 Sterne JAC, Sutton AJ, loannidis JPA, et al. Recommendations for examining and interpreting funnel plot asymmetry in meta-analyses of randomised controlled trials. BMJ 2011;343:d4002.

46 Cha J, Hwang J-M, Jo HJ, et al. Assessment of functional characteristics of amnestic mild cognitive impairment and Alzheimer's disease using various methods of resting-state fMR analysis. Biomed Res Int 2015;2015:1-12. 